\title{
154. Sur les Points Singuliers des Équations Différentielles Admettant un Invariant Intégral
}

\author{
Par Taro URA et Yoshikazu HIRAsAwa \\ Université de Kobe \\ (Comm. by K. Kunugi, M.J.A., Oct. 12, 1954)
}

\section{Introduction}

1. Dans une Note antérieure, un des auteurs a classé ${ }^{1)}$ les points singuliers de première espèce d'un système d'équations différentielles défini à la surface du tore sous la condition que le système admette un invariant intégral positif. On remarque cependant que cette classification est applicable, même si l'on supprime l'hypothèse faite que le système est défini à la surface du tore, puisque la classification des points singuliers est un problème local.

Nous proposons ici de considérer le cas, où l'on ne suppose pas que les points singuliers soient de première espèce, mais que l'on suppose seulement qu'ils sont isolés, et en modifiant la définition des cols, nous montrons que notre classification s'applique aux points singuliers d'un système d'équations qui admet un invariant intégral positif.

2. Aux paragraphes 3 et 4 , nous expliquons les hypothèses sur le système, et en particulier, précisons celles qu'un point singulier considéré est isolé et que le système admet un invariant intégral positif.

$\mathrm{Au}$ paragraphe 5, nous expliquons la notion de centres de Poincaré, et introduisons celle de cols généralisés; et à la fin de ce paragraphe nous énonçons un théorème à prouver, dont la démonstration se divise en trois parties.

D'abord, au paragraphe 6, nous montrons qu'un centre de Bendixson est nécessairement un centre de Poincaré dans le cas considéré. Donnant au paragraphe 7 la démonstration qu'il n'existe pas de régions nodales, nous montrons dans le paragraphe 8 qu'il n'existe qu'un nombre fini de caractéristiques aboutissant à un point singulier, si ce point n'est pas un centre de Bendixson.

\section{Hypothèses et Résultats}

3. Envisageons un système d'équations différentielles

$$
\frac{d x}{d t}=X(x, y), \quad \frac{d y}{d t}=Y(x, y),
$$


où $X(x, y)$ et $Y(x, y)$ sont deux fonctions continues des deux variables réelles $x$ et $y$, définies dans un voisinage $A$ d'un point $P_{0}=\left(x_{0}, y_{0}\right)$ du plan $(x, y)$, et $t$ est un paramètre représentant le temps.

Nous supposons que le point $P_{0}$ est un point singulier isolé, c'est-à-dire que le point $P_{0}$ satisfait aux équations

$$
X(x, y)=0, \quad Y(x, y)=0,
$$

et que, dans un certain voisinage $V$ du point $P_{0}$, il n'y a pas de solutions des équations (2) sauf le point $P_{0}$. Nous supposons que l'on a $V=A$, ce qui est loisible.

Supposons en outre qu'il existe une unique solution du système

$$
x=x\left(t, x_{1}, y_{1}\right), \quad y=y\left(t, x_{1}, y_{1}\right),
$$

qui remplisse les conditions initiales $x\left(0, x_{1}, y_{1}\right)=x_{1}, y\left(0, x_{1}, y_{1}\right)=y_{1}$, pourvu que le point $P=\left(x_{1}, y_{1}\right)$ appartienne à $A$. La courbe dans le plan $(x, y)$ représentée par la solution (3) sera appelée caractéristique suivant la terminologie de Poincaré, plus précisément, caractéristique passant par le point $P$ (à l'époque $t=0$ ), et la courbe partielle de la caractéristique correspondant aux valeurs $t \geq 0$ (ou $t \leq 0$ ) s'appelle demi-caractéristique partant du point $P$.

On voit, d'après cette définition, que la caractéristique passant par le point singulier $P_{0}$ se réduit au point $P_{0}$.

4. Supposons d'ailleurs que le système (1) admette un invariant intégral positif. Dire qu'une fonction $M(x, y)$ est un invariant intégral positif, c'est dire que $M(x, y)$ est uniforme et localement sommable dans $A$ et que l'on a

$$
0<M(x, y)<\infty \quad \text { presque partout dans } A \text {, }
$$

et enfin que, désignant par $U$ un sous-ensemble de $A$ quelconque, tel que $M(x, y)$ soit sommable dans $U$, on a

$$
\iint_{U(t)} M(x, y) d x d y=K
$$

pour toute valeur de $t$, pourvu que $U(t)$ soit défini, où $K$ désigne une constante indépendante du temps $t$, et où $U(t)$ est l'ensemble des positions à l'époque $t$ des points qui appartiennent à $U$ à l'époque $t=0$, et qui se meuvent selon le système (1).

5. Nous appelons ${ }^{2}$ le point $P_{0}$ centre de Poincaré, si toutes les caractéristiques assez proches du point $P_{0}$ sont périodiques, et le point $P_{0}$ est dit col généralisé, s'il n'existe qu'un nombre fini de caractéristiques aboutissant au point $P_{0}$ pour $t \rightarrow \infty$ ou $t \rightarrow-\infty$.

Avec les définitions précédentes, le théorème à démontrer s'exprime comme suit:

Théorème. Sous les conditions énoncées aux paragraphes 3 et 4 , le point singulier $P_{0}$ du système (1) est ou bien un centre de Poincaré, ou bien un col généralisé. 
Remarque. D'après un théorème de Bendixson, si l'on désigne par $n$ le nombre de caractéristiques aboutissant à un col généralisé pour $t \rightarrow \infty$ ou pour $t \rightarrow-\infty$, le nombre $n$ est pair, soit $2 m$, et il existe exactement $m$ caractéristiques aboutissant à ce col généralisé pour $t \rightarrow \infty$, et $m$ pour $t \rightarrow-\infty$.

\section{Démonstration du Théorème}

6. Dans un Mémoire, Bendixson a démontré ${ }^{3)} q u$ 'en considérant les caractéristiques assez proches du point singulier $P_{0}$, ou bien il existe une infinité de caractéristiques périodiques entourant le point $P_{0}$ (dans ce cas $P_{0}$ sera appelé centre de Bendixson), ou bien il existe au moins une caractéristique aboutissant à $P_{0}$.

Nous nous occupons d'abord du premier cas de l'alternative; nous supposons donc que le point singulier $P_{0}$ est un centre de Bendixson, et nous montrons que $P_{0}$ est un centre de Poincaré.

En effet, si $P_{0}$ n'était pas un centre de Poincaré, il y aurait dans un voisinage quelconque $V$ de $P_{0}$ une caractéristique nonpériodique $C$. Comme, par hypothèse, le point singulier $P_{0}$ est isolé, si $V$ est assez petit, la caractéristique $C$ s'approcherait, en figurant une spirale, d'une certaine caractéristique périodique $C_{1}$ pour $t \rightarrow \infty$ et d'une autre $C_{2}$ pour $t \rightarrow-\infty$. Sans restreindre la généralité, nous pouvons supposer que la caractéristique périodique $C_{1}$ est intérieure à $C_{2}$.

Soit $Q$ un point quelconque de $C_{1}$; désignons par $N$ un segment assez petit de la normale de la caractéristique $C_{1}$ au point $Q$, pour que toutes les caractéristiques passant par un point de $N$ ne soient pas tangentes à $N$. La caractéristique $C$ doit couper le segment $N$ en une infinité de points pour $t \rightarrow \infty$, et si l'on désigne par $P_{1}$ et $P_{2}$ deux points de cette intersection correspondant aux valeurs $t_{1}$ et $t_{2}$ de $t$ respectivement, telles que l'on ait $t_{1}<t_{2}, P_{2}$ est plus proche du point $Q$ que $P_{1}$. Supposons qu'il n'existe pas de point de cette intersection correspondant à une valeur de $t$ dans l'intervalle $t_{1}<t<t_{2}$, de sorte que $P_{2}$ soit ${ }^{4)}$ le conséquent du point $P_{1}$ selon la terminologie de Poincaré. On voit que cette hypothèse ne restreint pas la généralité.

Considérons la région fermée $U$ limitée par l'arc $\overparen{P}_{1}, P_{2}$ de $C$, par l'arc $\overparen{P}_{1}, P_{2}$ du segment $N$, et par la caractéristique périodique $C_{1}$; il est clair que $U$ contient $U\left(t_{2}-t_{1}\right)$ et que la mesure de $U$ est plus grande que celle de $U\left(t_{2}-t_{1}\right)$, de sorte que, $U$ étant compact, on a

$$
\iint_{U} M(x, y) d x d y>\iint_{U_{\left(t_{2}-t_{1}\right)}} M(x, y) d x d y,
$$


puisque par hypothèse on a $M(x, y)>0$ presque partout dans $A$; ceci est en contradiction avec (4). En conséquence l'hypothèse qu'il existe au moins une caractéristique non-périodique dans tout voisinage du $P_{0}$ est absurde, donc dans un certain voisinage du point $P_{0}$, toutes les caractéristiques sont périodiques, ce qui revient à dire, par définition, que le point singulier $P_{0}$ est un centre de Poincaré.

Remarque. Dans cette démonstration, la sommabilité locale de la fonction $M(x, y)$ au point singulier $P_{0}$ n'a pas été utilisée.

7. Ce point établi, passons au deuxième cas de l'alternative, et montrons d'abord qu'il n'existe pas de régions nodales aboutissant au point $P_{0}$.

Supposons en effet qu'il existe une région nodale aboutissant au point $P_{0}$ pour $t \rightarrow \infty$ (ou pour $t \rightarrow-\infty$ ). Soient $P$ un point de cette région nodale, et $C$ la demi-caractéristique partant du point $P$ et qui aboutit à $P_{0}$ pour $t \rightarrow \infty$ (ou pour $t \rightarrow-\infty$ ). Considérons la normale de $C$ au point $P$, et désignons par $N$ un petit segment de cette normale, dont le point $P$ est une des extrémités. On peut supposer que le segment $N$ est assez limité, pour que $N$ soit contenu dans la région nodale considérée et que toutes les caractéristiques passant par un point de $N$ ne soient pas tangentes à $N$; toutes les caractéristiques passant par un des points de $N$ aboutissent à $P_{0}$ pour $t \rightarrow \infty$ (ou pour $t \rightarrow-\infty$ ). Soient $Q$ un point de $N$ distinct de $P$, et $C_{1}$ la demi-caractéristique partant de $Q$ qui aboutit au point $P_{0}$ pour $t \rightarrow \infty$ (ou pour $t \rightarrow-\infty$ ); la région $U$ limitée par les deux demi-caractéristiques $C$ et $C_{1}$, par le segment partiel $\overparen{P, Q}$ de $N$ et par le point $P_{0}$ contient la région $U(t)$, et par ailleurs la mesure de $U$ est plus grande que celle de $U(t)$ pour toutes les valeurs de $t$ positives (ou négatives). Ainsi, puisque $U$ est manifestement compact, en raisonnant comme au paragraphe précédent, il résulte une contradiction avec l'hypothèse que le système admet un invariant intégral positif.

En conséquence, l'hypothèse d'existence de régions nodales est absurde.

8. Nous disons maintenant qu'il n'existe qu'un nombre fini de caractéristiques aboutissant au point $P_{0}$. Pour démontrer cette proposition, supposons qu'il existe une infinité de pareilles caractéristiques, et montrons que ceci conduit en contradiction.

En effet, s'il existait une infinité de caractéristiques aboutissant au point $P_{0}$ pour $t \rightarrow \infty$, nous pourrions décrire un cercle $\Gamma$ entourant le point $P_{0}$, tel que, tendant vers le point $P_{0}$ pour $t \rightarrow \infty$, une infinité de caractéristiques coupe le cercle $\Gamma$. Désignons par $E$ l'ensemble des points en lesquels les caractéristiques aboutissant 
au point $P_{0}$ coupent le cercle $\Gamma$ pour la dernière fois; l'ensemble des points d'accumulation de $E$ est non-vide et contenu dans $\Gamma$, puisque $\Gamma$ est compact. En outre, en raison de la continuité des caractéristiques par rapport aux points initiaux, on voit aisément qu'au point $P_{0}$ aboutit la demi-caractéristique $C$ partant d'un de ces points d'accumulation, soit $Q$, pour $t \rightarrow \infty$.

Envisageons la normale de la demi-caractéristique $C$ au point $Q$ et limitons cette normale à un segment assez petit, soit $N$, pour que toutes les caractéristiques passant par un point de $N$ ne soient pas tangentes à $N$.

Le point $Q$ étant un point d'accumulation de $E$, il existe des points de $E$ aussi proches du point $Q$, que l'on veut; donc, à cause de la continuité des caractéristiques, il existe ${ }^{5)}$ un point $P$ de $E$, tel que la caractéristique $C_{1}$ passant par le point $P$ coupe le segment $N$ en un point $P_{1}$. Comme la caractéristique $C_{1}$ aboutit au point $P_{0}$ pour $t \rightarrow \infty$, on peut supposer qu'après le point $P_{1}$, la caractéristique $C_{1}$ ne coupe plus le segment $N$. Sans aucune ambiguité, nous pouvons désigner par $C_{1}$ la demi-caractéristique partant du point $P_{1}$ et aboutissant à $P_{0}$.

Considérons la région limitée par les deux demi-caractéristiques

$C$ et $C_{1}$, par le segment partiel $\overparen{P_{1}, Q}$ de $N$ et par le point $P_{0}$; par cette construction, $U$ appartient visiblement à une certaine région nodale, ce qui est en contradiction avec le résultat du paragraphe précédent.

Donc il n'existe qu'un nombre fini de caractéristiques aboutissant au point $P_{0}$ pour $t \rightarrow \infty$, et de même on démontre la même proposition concernant les caractéristiques aboutissant au point $P_{0}$ pour $t \rightarrow-\infty$.

En conséquence le point singulier $P_{0}$ est un col généralisé, ce qui achève, avec le résultat du paragraphe 6 , la démonstration $d u$ théorème énoncé au paragraphe 5 .

\section{Références}

1) T. Ura: "Sur les courbes définies à la surface du tore par des équations admettant un invariant intégral ", Ann. Ec. Norm., [3], 69, 260-263 (1952).

2) H. Poincaré: “Mémoire sur les courbes définies par une équation différentielle”, J. Math. pures et appl. [3], 7, 390 (1881); OEuvres I, 17 (1928).

3) I. Bendixson: "Sur les courbes définies par les équations différentielles", Acta Math., 24, 26-27 (1901). E. Kamke: "Differentialgleichungen reeller Funktionen ", 222-223 (1930).

4) H. Poincaré: “Mémoire sur les courbes définies par une équation différentielle", J. Math. pures et appl. [3], 7, 253 (1881); CEuvres I, 46 (1928).

5) T. Ura: “Sur les courbes définies par les équations différentielles dans l'espace à $m$ dimensions ", Ann. Ec. Norm., [3], 70, 303-304 (1953). 